\title{
Protée
}

\section{Gisement pour la singularité}

\section{Francesca Caruana}

Volume 30, numéro 3, hiver 2002

Autour de Peirce : poésie et clinique

URI : https://id.erudit.org/iderudit/006867ar

DOI : https://doi.org/10.7202/006867ar

Aller au sommaire du numéro

\section{Éditeur(s)}

Département des arts et lettres - Université du Québec à Chicoutimi

\section{ISSN}

0300-3523 (imprimé)

1708-2307 (numérique)

Découvrir la revue

\section{Citer cet article}

Caruana, F. (2002). Gisement pour la singularité. Protée, 30(3), 45-54. https://doi.org/10.7202/006867ar

\section{Résumé de l'article}

$\mathrm{Au}$ contact d'œuvres d'art, nous sommes enclins le plus souvent à interroger les significations possibles contenues dans les sujets représentés. La question se pose de savoir comment ces sujets, fussent-ils non figuratifs et adoptant de multiples formes de présentation, résorbent la nécessité d'être exprimés. La sémiotique de Peirce fournit des éléments de compréhension lorsqu'on aborde le processus de création. Il est lié aux surgissements de signes, empreintes corporelles originaires de notre organisation psychique, qui sont eux-mêmes travaillés par le musement, flot ininterrompu de nos pensées. L'inscription produite dans le moment de création est une rupture du musement " esthétique ", rupture dont le caractère temporel dénote une importance identique à celle vécue dans la cure. En ce sens, l'inscription est première et fait retour sur un principe associatif inconscient, disponible hic et nunc. Ce principe lui est second puisque émergé par coupure dont la continuité s'exerce en révélant singularités et idiolectes plastiques.
Ce document est protégé par la loi sur le droit d'auteur. L'utilisation des services d’Érudit (y compris la reproduction) est assujettie à sa politique d'utilisation que vous pouvez consulter en ligne.

https://apropos.erudit.org/fr/usagers/politique-dutilisation/ 


\section{GISEMENT POUR LA SINGULARITÉ}

FRANCESCA CARUANA

Il serait légitime de s'interroger sur ce qu'une artiste, fût-elle aussi sémioticienne, pourrait avoir à dire sur le rapport entre art et psychanalyse, si ellemême, depuis toujours, n'interrogeait pas le processus de création, la singularité qui en gouverne les modalités et la temporalité du corps qui crée.

L'article qui suit se fonde sur l'expérience de l'atelier, mais aussi sur le fait que l'œuvre produite n'appartient pas en totalité au créateur, que l'inscription plastique réalisée est de l'ordre d'un idiolecte. On peut alors saisir que le contexte vital de l'artiste, bien au-delà des éléments biographiques, «travaille» le processus créateur et rend possible un objet dont les "pairs» iront jusqu'à le classer «art» ou pas ${ }^{1}$. C'est l'idée même de cette intrication, entre l'être au monde, entre l'espace et la forme, qui supporte l'ensemble de mes propos.

\section{LES LIMITES}

Il y a de nombreuses années, une orthophoniste me signala l'existence des "gouttelettes de Pflugger " ${ }^{2}$. C'est une image qui m'avait frappée car elle délimitait un espace corporel, du corps existant, que chacun fait respecter, que ce soit sur le plan de la distance à tenir dans les conversations, ou quant aux codes de bienséance qui légifèrent sur la distance à adopter entre personnes. Il s'agit de cette aura de buée, de cette qualité d'haleine qui n'a rien à voir avec les postillons, qui se dégage de chacun d'entre nous et qui nous donne la température ou l'odeur de l'autre. Plus on s'élève dans la hiérarchie sociale, plus la distance à tenir est grande et met de l'espace entre les corps. Quel enjeu ou quel danger représenterait la proximité des corps? Se souillerait-on de la vapeur de l'autre?

Quoi qu'il en soit, cette limite intégrée dans le système de communication fait que chacun dispose d'un espace inscrit par l'habitude, qu'il gère de manière «spontanée» en respectant et faisant respecter cette distance en deçà de laquelle la situation est intenable. Seuls certains artistes comme les danseurs ou les comédiens transgressent cet espace, espace que le metteur en scène a conçu comme perméable à la présence simultanée de deux haleines, dans le cas où les deux corps sont considérés comme un seul ou lorsque le sens du texte acquiert une intensité plus grande lorsqu'il est dit «dans» la bouche de l'autre. Il est intéressant de noter que le 
respect de la distance est une règle absolue dans la peinture traditionnelle. Même un peintre comme Goya, qui a transgressé certaines règles de représentation et de protocole, soumet ses personnages à un représentation de face, quelle que soit leur situation d'intimité, comme s'il s'agissait de la désamorcer en la présentant tout en se préservant d'une certaine indécence ${ }^{3}$. C'est bien de cela dont il est question, ce qui touche à la proximité des corps. L'espace des gouttelettes organise les limites de la décence. Ou de l'indécence.

L'artiste, je crois, est cet être qui n'a pas résolu cette rupture obligée d'avec le corps «large» auquel il appartient. Le leurre est de penser que le corps est individuel, avec sa propre existence (ce que l'on peut dire de la délimitation par la surface corporelle). Le fantasme de l'indépendance. Lors d'un accident, c'est mon corps, bien sûr, qui est atteint et pas celui du voisin. Toutefois, cette pensée du corps est autre chose qu'une découpe dans la masse plus grande d'un corps «social». Ce corps-là est un corps second, accidentel, et doublement puisque accidenté. Il est à concevoir comme ayant une existence "générale», comme on peut le penser pour l'eau: elle a une existence «générale» qui n'est pas celle d'une existence singulière lorsqu'elle coule du robinet, elle est différenciée en cela de l'eau du torrent ou de la mer.

\section{LES EFFETS DU CORPS}

Les intrications que l'on note, entre ce que l'on peut considérer comme la masse humaine et l'homme, dès que l'on s'intéresse au processus de création, révèlent que les formes produites par le créateur portent les traces de ce corps social dont il n'a pas réussi à symboliser les limites. Il s'agirait d'une sorte de désir inconscient de réactualiser la fusion originelle, de rendre compte du magma initial, de résorber une inquiétude de séparation. Pour ceux qui n'ont pas cette résistance à la symbolisation ou à la convention établie par le corps social, la vie est fluide, l'attitude dyadique, en réponse aux projets immédiats que proposent les contraintes sociales de déplacement, de réflexion, de travail, d'imaginaire, etc. En fait, la résistance à cette séparation que la première limite, concrétisée par les «gouttelettes de Pflugger", donne à l'existence, inscrit chez certains êtres une obligation à sortir du dilemme par la création. C'est de ce malaise, par rapport à l'idée de limites qui «individuerait» l'être, que l'artiste fait une rupture et que, paradoxalement, il tente de rétablir un lien de continuité avec le corps large qui lui échappe.

Quand l'artiste est au travail, à un moment donné quelque chose "prend", qu'on peut qualifier de cristallisation. La question est de savoir quelle est la nature de cette matière qui prend et affirmer ou pas si elle appartient à un processus. En tant que plasticienne, $\mathrm{j}$ 'ai tendance à répondre par l'affirmative, dans la mesure où nous ne partons pas d'ex nihilo et que le moment où «ça prend» est malgré tout un moment conclusif. L'idée de processus pourrait être remise en cause si nous n'avions pas la possibilité (et à la condition) de distinguer les sémioses entre elles ${ }^{4}$. Le premier niveau d'interprétation de la sémiose de création est précisément de reconnaître que "ça prend", sans savoir pourquoi et sans conscience d'être une manière de stopper le savoir. Il s'agit d'une sémiose dégénérée de la sémiose de création ${ }^{5}$. Pour tenter de répondre à la question de savoir comment qualifier la cristallisation (ce moment où l'œuvre prend), je me rends compte, en décrivant un «bâton de deuil» réalisé pour une exposition ${ }^{6}$, que, finalement, quand "ça» prend, cela n'a rien à voir avec un savoir, mais avec une richesse insue, un trésor qui est en amont de la création, qui a travaillé le corps et duquel un objet se révèle, prend forme. On pourrait dire, à la suite de cette approche, que tout objet produit (en dehors de toute considération de sujet) est la résolution d'une question relative à l'espace, dont il est une métaphorisation.

\section{LA QUESTION DES TESSĖRES}

Lorsque l'œuvre prend, cela se fait, indépendamment de toute pensée, de toute intention, par l'arrêt brutal du processus créatif alors que les formes mêmes par lesquelles il se manifeste 
(psychique, matérielle, culturelle, etc.) incitaient à la continuité créatrice. L'artiste, sans l'avoir consciemment "décidé ", met un terme, physique, réel à la peinture ou à la construction du montage. Le rapport du scribe au museur s'arrête là. Il y a un premier niveau de l'interprétation de la sémiose de création qui peut dire "c'est fait, c'est pris", c'est conclusif de cette sémiose-là. Le «savoir» impliqué dans la procédure, par laquelle l'artiste s'arrête de composer, attaché à ce moment où les choses prennent, tient lieu de tessères corporelles ${ }^{7}$. Ces tessères rétablissent la continuité du signe entre les œuvres que l'on contemple et les œuvres en train de se faire. Le cas privilégié de l'observation des enfants montre bien qu'il s'agit de tessères corporelles et que l'artiste est celui qui dégage une résistance à cet espace balisé et conventionnel qui lui a été signifié dès sa petite enfance. Quand un enfant entasse des cubes troués sur un bâton, il fait preuve de création, il s'installe dans tout espace sans avoir conscience de transgresser l'espace de l'autre, il vit la notion d'espace «naturel», il peut faire ses entassements sans que quiconque ne trouve à redire. Pourtant, si un adulte faisait la même chose, il aurait toutes les chances d'être qualifié de bizarre, de maniaque, etc. Ce qui fait limite ici est la notion même de création, intimement liée à celle du corps; et l'histoire des gouttelettes ne peut se comprendre qu'en voyant dans l'acte de création le désir de relier, de réparer le corps large auquel l'artiste se sait appartenir, mais dans lequel il est séparé par l'organisation sociale. Il refait les gestes de l'enfant car, dans le lieu «art» où les suspicions de bizarreries sont anticipées, la société autorise les individus à rétablir la voie des tessères corporelles, seul lieu où les symboles et les inhibitions ont la chance de ne pas se perpétrer. Ainsi, quand l'artiste sent sa pièce "prise» et qu'il peut mettre le mot fin, c'est un premier niveau de l'interprétation de la sémiose de création, mais c'est aussi l'inscription des tessères corporelles qui viennent, de manière iconique, prendre la place de ce qui, dans d'autres pratiques sociales, est généralisé, symbolisé, balisé, codifié. Les tessères corporelles seraient «l'explication» de la question de la cristallisation: ce mot «fin» que chaque artiste met à son œuvre, à son insu.

Concernant le processus de création, il s'avère complexe de citer en exemple l'œuvre de tel ou tel artiste - l'œuvre en elle-même n'étant pas une illustration-type des tessères corporelles, mais l'image du processus qui conduit l'acte de création. Tout artiste est pourtant concerné par cette organisation créatrice. On peut cependant désigner l'exemplarité de l'œuvre de Joseph Beuys d'un double point de vue: d'abord par des «déterminations» originaires qui l'ont fait artiste et pas autre chose; ensuite, par des événements de sa vie au travers desquels cette économie d'empreintes originaires a été maintenue. En effet, répétons-le, comme pour tout artiste, si l'on ignore la raison pour laquelle un individu est artiste, ce sont les effets produits par lui qui nous renseignent sur l'art. Ainsi, avant l'écrasement de l'avion dans lequel il avait pris place, en Crimée, en 1943, et qui eut des incidences sur sa technique artistique, Beuys exécutait des dessins dont le thème et le soin apporté au choix des objets montraient un réel souci d'expression d'une chose intérieure, qu'il appelle «l'Âme». Si rien ne nous permet encore de connaître l'instant où ces traces originaires se forment au cours de l'histoire personnelle (en l'occurrence un ensemble de représentations - animaux, feuilles...), tout dans l'œuvre de Beuys laisse penser que se sont fixées en lui des inscriptions, de nature à resurgir sous la forme créatrice "d'empreintes», supportées par des «matériaux»:

Il n'existe pas d'autre possibilité de s'exprimer que par un signe d'un matériau déterminé. Et pour cela, on a besoin de matériaux plus ou moins solides. [...] Car les hommes ne peuvent s'exprimer qu'à travers des formes laissées par des matériaux déterminés. Cela est bien sûr également vrai pour la langue. ${ }^{8}$

Beuys explicite, par la nécessité de l'expression, la présence de tessères corporelles sans cesse pétries par le musement. Ainsi, lors de l'accident de 1943, Beuys fut sauvé par la population tartare qui l'enveloppa de graisse et le protégea avec du feutre. Cette expérience lui donna des idées qu'il expérimenta pendant 
plusieurs années dans sa production plastique. La forme concrète que prit alors le musement se caractérisa par une nouvelle inscription plastique de ses travaux. Il réalisa des œuvres

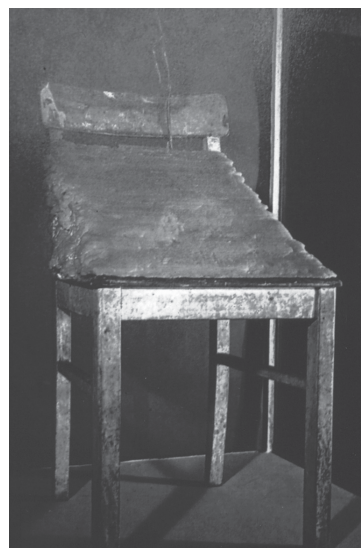
que Infiltration homogène pour piano à queue (1966) et Chaise avec graisse (1963). Chaque ouvre fut un mot «fin", une rupture dans le musement de l'artiste. On pourrait aller jusqu'à dire que le terme "fin" apposé à chaque ouvre rend compte de la solidification d'une image vague, révélée du musement par son inscription.

Néanmoins, il faut différencier ce mot «fin» de celui d'un interprète qualifié. Ici, il est question du corps, dans son expression de l'intime, et il est nécessaire d'établir une distinction entre la réparation de l'artiste et l'attitude commune. Cette dernière ne fait pas état d'une singularité, puisque tout le monde réagit et possède ce mouvement de recul devant l'espace du visage; en revanche, les solutions que les artistes trouvent pour effacer ou atténuer ce «malaise de séparation" sont les traces portées par le processus de création. Effectivement, s'il n'y avait pas de conclusion à la sémiose de création, alors on serait dans la répétition incessante des formes, puisque l'œuvre ne prendrait jamais. Je crois aussi, en ce sens, que la fin de l'art n'est pas seulement la délectation, comme le disait Poussin, mais également l'imitation. Il faut entendre: le désir d'imiter, et non pas le sujet de l'imitation, qui est ici sans importance. Il y a dans l'imitation les éléments mortifères propres à l'autoengendrement qui, sans altérité, rejoint la recherche d'unicité contenue dans la pulsion de mort.

\section{LOGIQUE DES TESSÈRES}

Ces tessères tiennent lieu de signifiants car elles sont mises pour un autre signifiant. Mais la question

reste difficile car on aura du mal à affirmer qu'en tant que signifiants, ce sont des légisignes, puisqu'elles sont secondes. Concernant le corps, ce sont des «signifiants »-traces, non symbolisés. La question demeure de savoir si cette notion hybride a quelque validité que ce soit. Tout se passe comme si ces tessères organisaient le sujet du corps. Un double sujet ou un sujet divisé, dans la mesure où l'œuvre nous propose une "double structure divisée», comme le dit M. Fried. La fiction du sujet et la forme qui ouvre sur un monde qui ne se veut pas être ce qu'il est; la fiction qui s'oppose à la planéité du tableau et qui ouvre sur un monde qui ne se veut pas plat.

L'artiste serait donc celui qui, par le processus de réparation, restaure le sujet, lui rend une certaine unité; mais cette unité n'est redevable qu'à la fiction produite par l'exigence des tessères corporelles. De cet inconfort permanent, l'œuvre est produite.

Les tessères n'ont pas de formes préalables, elles sont improvisées et révélées par le sujet qui répond à des manques ou à des pertes, eux-mêmes source d'angoisse créée par la discontinuité formelle et sociale. Il faut savoir que toute convention s'appuie sur une égalisation du plaisir non contrôlé et en produit l'anéantissement. Ainsi la régulation sociologique contenue dans le savoir-vivre tend à répondre à ce type de programmation d'une loi corporelle, naturellement dissoute dans l'éducation, à savoir que là encore l'artiste ou le marginal transgresse sans sourciller, mais bouleverse l'ordre de séparation. On peut, à ce terme, chercher à savoir s'il s'agit ici de séparation ou de réparation, puisque, dans un tel cas, l'une naît de l'autre.

Cette question nous conduit à examiner le vecteur principal de ces investissements, le musement.

\section{À PROPOS DU MUSEMENT OU UN ARGUMENT EN FAVEUR DE LA RIGUEUR D'INTERPRÉTATION La notion de continuité dans le domaine de la création \\ Nantis de cette compréhension de nos propres modes d'être par le musement, il est aisé d'envisager l'idée d'une continuité ininterrompue de pensées, de sensations, de perceptions. C'est dans ce continuum}


qu'intervient une rupture, une découpe qui tient lieu à un moment donné de représentation, d'un état des lieux de l'état interne, de la marmite en quelque sorte. La somme des ingrédients est ce que la marmite peut contenir. Notre musement, fait de bords et d'ingrédients informes, bouillonne en tous sens, jusqu'à ce qu'une rupture, un appel, une idée, un geste ou un bruit fasse irruption et délivre quelque chose (on devrait dire calque-chose, «image" produite de cette sève et du contexte dans lequel l'événement s'est produit, imprégné de son lieu d'origine et par sa source). Cette chose-là est contextualisée, même si les apparences n'apportent pas de preuve. Beuys, passant dans un marché, peut ne pas être sensible à une qualité de feutre vendu au mètre, sans le voir, mais ce passage pourra tout aussi bien provoquer en lui l'idée d'une installation où les murs seront complètement recouverts par ce matériau, conjonction de matière, sensation, pensée, avec leurs conséquences, c'est-à-dire cette priméité de la tiercéité qui rend compte des qualités du consensus auquel chacun de nous appartient: notre registre et ses entrailles.

\section{Secondéité du musement}

Il reste que toute irruption dans le musement, pour hasardeux qu'il soit, n'en provoque pas moins une découpe. Cette découpe a une identité dans le continuum magmatique et infini de nos possibles. C'est une découpe hic et nunc qui se fait et, malgré tout, elle n'est pas n'importe laquelle. Lorsqu'une personne muse, il va de soi que, parmi tous les possibles, tous ne sont pas présents en même temps, que le musement est un objet insu de notre appareillage inconscient, de sorte que la découpe fait apparaitre une sélection a priori, et qu'elle révèle un aspect «inconscient» a posteriori, lorsque le "retour sur» le musement intervient comme un lapsus et nous permet de dire ce qu'il était.

On constate ainsi que tout de notre esprit n'est pas disponible en même temps: il y a une sélection d'images et de "pensées" qui irriguent hic et nunc le flot. L'ensemble demeure un possible (une priméité) à disposition, mais non requis à ce moment-là. C'est le caractère temporel du musement qui implique le fait qu'il a une certaine identité. Son identité est contextuelle. Pouvoir y revenir (le «retour sur») est la tiercéité du musement. Étant un signe de priméité, ses interprétants sont iconiques, ce qui n'est pas sans importance pour l'artiste, qui n'est pas acharné à interpréter ses œuvres.

\section{Conséquences pour le processus créateur}

Le processus de création fait apparaître au monde un objet dont l'artiste ne sait pas ce qu'il est. Ce que l'artiste sait, c'est qu'il le fait ici et maintenant, "poussé» par une énergie interne, un tonus, qui met au jour très approximativement ce qui est «musé». L'objet de création est ignoré et exprime une forme de singularité. D'où la diversité des propositions et les dangers inhérents au style. Le musement ancré dans les signes diacritiques d'un style quel qu'il soit est une sorte de danger pour la création. On pourrait faire ainsi l'hypothèse que les artistes qualifiés de «suiveurs» sont ceux-là même dont le musement est envahi par les indices d'une garantie d'art, tels qu'on les repère dans la notion de style.

L'art est ainsi régulièrement confronté à la sacralisation de la sensibilité de l'artiste, à l'interprétation, à la spontanéité avec laquelle les œuvres ont été produites. L'art n'a pour effet que de constituer un préjudice: artistes et œuvres ne peuvent que «pâtir» de ce qui sera dit à leur sujet, car, précisément, tout se passe comme si la secondéité du musement était ignorée et que le musement dans son acception totale de priméité était présent tout entier (il ne s'agit pas pour autant d'en déduire qu'il y a une interprétation orthodoxe dont l'œuvre ne souffrirait pas, sous couvert d'unilatéralité de l'interprétation; ce dont il est question, c'est que de toute œuvre, toute interprétation possible est contenue dans ce qu'elle livre, mais pas au-delà, parfois en deçà). Dans son ouvrage consacré à Giorgione, S. Settis fait la preuve des propositions multiples affectées à une œuvre, où la validité des unes renvoie à la possible vérité de l'autre: le facteur discriminant est la mesure même donnée par les signes plastiques ${ }^{9}$. On ne pourra 
jamais dire qu'Arcimboldo était un précurseur du surréalisme parce que ce terme même n'était pas conçu. Les possibilités d'un tel mode de représentation fondé sur un principe associatif irrationnel n'était pas concevable, à ce moment-là; du point de vue des signes plastiques, la présence de légumes ou de fruits n'étaient que des objets mis pour d'autres, mais dont la signification n'avait aucune ambiguité.

La difficulté de l'interprétation de toute œuvre comme œuvre d'art rejoint celle de certains malades mentaux, ou de blessés qui ont subi un syndrome frontal et qui pourtant, grâce à l'originalité et à la persévérance de soins particuliers, maintiennent une fonction d'écriture ${ }^{10}$. Comment interpréter ces écritures qui sont plus iconiques que scripturales?

\section{ANALOGIE POUR L'INTERPRÉTATION DU MUSEMENT}

Est-ce que leur écriture ne serait pas aussi de l'ordre d'une secondéité du musement? Rendrait-elle compte de ce caractère iconique de l'écriture ellemême (elle ressemble complètement à la cursive habituelle, prélevée, découpée, dans le continuum du musement)? Ainsi ce type d'écriture n'introduirait aucune discontinuité dans le musement du patient, il s'agirait d'une rupture sans abduction mais qui viendrait seulement le prolonger. C'est ce que l'on constate d'ailleurs chez les artistes: l'œuvre prolonge le musement, mais elle est «brouillée» par son inscription sociologique, qui intervient $a$ posteriori sur le musement par abduction. On peut alors se poser la question de savoir dans quel contexte cette notion apparaît, et s'apercevoir que Peirce en parle à propos de la réalité de Dieu ${ }^{11}$. De ce point de vue, c'est une forme historique de la pensée.

En effet, comment justifier, si ce n'est par une nécessité conceptuelle du contexte historique, d'une place attribuable à Dieu, après le cartésianisme et la rigueur kantienne? Le pragmatisme de Peirce peut avoir aussi opté pour le fait que, puisque l'idée de Dieu existe, autant la classer dans un type de réalité qui est la réalité des possibles. Cette remarque n'a d'intérêt qu'en ce qu'elle permet de considérer cette secondéité comme dissociée de l'existence; sur ce plan, elle représente non plus les catégories de l'expérience, mais celle de l'obsistence. Il y a une secondéité et la secondéité, l'opérateur les distingue dans des domaines de perception du monde différents ${ }^{12}$. La vérité est «invariablement produite exactement comme l'argument négligé la produit» "13. De conséquent à antécédent, le processus montre la réalité de l'abduction ou plutôt celle d'une rétroduction ${ }^{14}$. Ainsi, ce qui relève de l'art spontané, livré à l'interprétation sauvage du sentiment, n'est qu'une illusion, une fiction concernant une dé-contextualisation du musement.

\section{IMPORTANCE DU PRINCIPE ASSOCIATIF DANS L'INTERPRÉTATION}

Si on a, depuis le concept de musement, opté pour un flot continue de pensées et pour son maintien en tous lieux et en toutes circonstances, je suis tentée aujourd'hui de mettre en évidence la secondéité du musement et la logique de ce qu'il nous donne à "voir», pour établir le caractère essentiel du principe associatif dans l'interprétation de l'art.

Le concept de musement développé par Peirce est une sorte de totalité première de notre mode d'être au monde. En cela, il est continu, et comme l'a montré M. Balat par l'hypothèse de cette continuité dans le cas des phases végétatives de l'éveil de coma, il est cette nappe commune aux êtres, enrichi de l'expérience individuelle et des savoirs anciens.

Si je souhaite insister sur la secondéité du musement, c'est que le moment précis où nous réalisons que nous avons musé est un moment de rupture, celui au cours duquel nous faisons un retour sur lui. Nous croisons quelqu'un dans la rue, puis nous nous arrêtons dix pas plus loin: la reconnaissance de la personne croisée est une rupture dans le musement, qui le signale et qui a une identité.

C'est de cette identité dont je voudrais dire qu'elle est seconde. Le passant a bien été perçu, mais au moment où le corps tout entier percevait, il était indisponible pour cette découpe interprétative. Quelles que soient les images formées, elles étaient 
une forme de sélection de ce que le corps est, ou de ce qu'il a en mémoire. À ce moment-là, c'est une certaine qualité d'images qui émergeait à cette préconscience et pas d'autres. Or il ne s'agit pas de mémoire à proprement parler - qui, elle, mobilise l'intellect -, mais d'un effet d'émergence qui «contenait» cela et autre chose, là, maintenant. Car l'ensemble de ce qui nous constitue est là, mémoire ou pas, et nous ne savons pas pourquoi nous associons cette image avec telle autre, comment ces flots de pensées, ces visualisations intérieures s'articulent entre elles, et si même elles se forment selon une perception visuelle, sonore, tactile.

Certes la perception est le lieu de tous les tremplins possibles pour le musement, mais il y a sans doute aussi des articulations propres à la logique interne du musement, et c'est en ce sens qu'il est une «représentation» de l'inconscient. La nature de l'inconscient passe peut-être par ces ligatures incompréhensibles à la gratuité apparente, par ces associations de termes pris entre événements extérieurs, même microscopiques, et notre effort constant à être un vivant. La place de la mémoire est donc mise en difficulté puisqu'on accède au musement par rupture, mais, dans cette temporalité, c'est un certain flot de pensées qui occupait l'esprit et pas le tout du musement. Or ce tout est disponible et opératoire tout le temps, pétri par les modifications quotidiennes de l'expérience, du savoir, de la douleur, des projets, etc. Si le musement relie des pensées entre elles, sans guide, comme le feraient les hasards d'une errance, elles sont le hic et nunc du musement qui en caractérise la secondéité et qui fait de ces pensées-là, une fois la coupure opérée par le retour sur elles, une secondéité de priméité. Cette dichotomie n'aurait pas d'intérêt particulier si elle ne mettait en évidence la coupure comme événement fondamental - elle fonctionne comme un lapsus pour le musement. Ce surgissement soudain permet un accès à ce qui est ignoré et fonctionne selon le modèle de l'insconscient, en pointant ce que le corps oublie, refoule. La sélection des images se cristallise sur certains points et il y a une absolue nécessité à ce que la cure fonctionne par associations pour retrouver cette nature du sujet qui muse. Le volcanisme du sujet qui produit un signifiant dans la cure rétablit une ligature d'une autre espèce, réparatrice celle-là, mais dans la configuration singulière du patient, sans aucune autre validité que pour lui-même. C'est ce que l'on peut dire de l'interprétation «idéale» d'une œuvre.

\section{MUSEMENT, MÉMOIRE, ASSOCIATION, INCONSCIENT}

Le principe associatif présente par analogie les aspects du fonctionnement de la pensée. À l'inverse, une méthode préalable ne laisse que peu de chances à l'hypothèse et, en ce sens, la sémiotique est un outil et non une science. À la lumière du rapport établi entre la secondéité du musement et ces images reproduites inconsciemment, qui elles-mêmes renvoient soit à une pathologie soit à une résolution, le terme de sélection apparaît contradictoire de celui d'association. Il n'en est rien. Et pour cela il faut faire appel à la logique. C'est une logique du musement, de notre manière d'être au monde et de notre savoir: leur intrication produit un certain type d'associations qui fait la richesse de l'hypothèse ou la possibilité d'élargir l'interprétation traditionnelle.

La notion de style, certaines concomitances scientifiques, les convergences esthétiques entre Braque et Picasso, les «coïncidences» ordinaires - avouant "avoir eu la même idée que» -, tout cela ne montre pas une matière commune, celle d'un musement "collectif»; ce qui revient à dire qu'il agit sur le plan d'une priméité travaillée.

Le musement apparait comme un concept fondateur: étant la possibilité de tous les possibles, il crée de l'impossible par exclusion de toute pensée impossible et fait la preuve que le processus créateur n'est que ce qu'il peut être; il évacue la sublimation, l'idéalisation et la surenchère dédiées traditionnellement à la notion d'œuvre.

Si la singularité est l'opérateur absolu du processus créateur, les termes de l'idiolecte plastique qui surgit sont tissés d'objets communs de pensée, d'humus constamment soulevé par les propositions du champ 
de l'art et d'une appartenance insue qui pétrit les modalités de l'expression.

\section{TEMPORALITÉ DU MUSEMENT}

En abordant la question de la singularité, la notion de temporalité s'impose. Un temps conjugué à l'espace, celui du présent, de l'être-là, lorsqu'il est impossible d'envisager des calculs pour la création. Il y a une interdépendance entre le musement, l'espace et le temps de l'expression. Tout comme dans une séance d'analyse. De même qu'il est impossible de penser que le patient parlera sous le nez de l'analyste, de même l'espace qui lui appartient est celui autorisé par l'espace du cabinet. Il ne peut pas non plus parler à la fenêtre; sans le savoir, le patient cherche à ce que sa parole «prenne» - comme une œuvre qui fabrique, élabore - pour que se produise ce qu'il ignore, c'est-àdire le surgissement d'une forme qui annulera, pansera, modifiera le nœud conflictuel. Il va sans dire que ce quelque chose, là, surgissant, n'est pas lié à une signification. Il est. La signification s'imposera du fait de le reconnaître comme terme signifiant et plastique selon le même processus décrit par Lacan:

Le signifiant est à concevoir comme distinct de la signification. Ce qui le distingue, c'est d'être en lui-même sans signification propre. Essayez d'imaginer dès lors ce que peut être l'apparition d'un pur signifiant. Bien entendu, nous ne pouvons même l'imaginer, par définition. Et pourtant puisque nous nous posons des questions d'origine, il faut tout de même tenter d'approcher ce que cela peut représenter. 15

Non seulement le processus de création en est la démonstration, mais la similitude est frappante lorsqu'on imagine la parole vacante, parfois à peine audible (d'ailleurs on vérifie toujours que le psychanalyste écoute, qu'il suit bien notre narration! Or c'est une réelle absurdité car, à cette question, nous savons qu'il écoute toujours, il muse son écoute et permet ainsi à notre musement de vaquer à son développement hasardeux) et qui, brusquement, est «entendue» par l'Autre, par un être transféré, le temps d'une prise, d'un encollage de types, ou de signifiants, dirait-on en termes lacaniens ${ }^{16}$.
Ainsi, on peut être affolé par la houle qui anime la question, par les multiples entrées, par les sources tentaculaires, dont l'écriture descriptive, elle-même en cet instant, est encore une image du musement (même si une certaine maîtrise ou une certaine orientation y sont engagées).

\section{L'IDIOLECTE PLASTIQUE}

S'il doit exister un lien entre les fonctionnements de la psychanalyse et le processus créateur, il est, à n'en pas douter, contenu dans les qualités de sentiments propres à rendre compte de signes singuliers, ceux qui débordent du cadre, de la coloration, de la composition ou encore de la convention, pour ne pas dire du sujet. On voit bien par l'ambiguité des termes que la relation s'installe d'elle-même. Lorsque l'art se limite au cadre, aux couleurs, au sujet, il y a fort peu de chances que, de nos jours, l'œuvre soit une création; je veux dire en cela que le processus créateur n'est pas à l'œuvre, l'objet produit n'est rien d'autre qu'un des effets de l'imitation, il n'est qu'un respect des codes plastiques traditionnels qui travaillent l'artiste et induisent une conformité. L'œuvre à ce moment-là pourra être vérifiée comme œuvre, elle en aura tous les signes diacritiques, mis à part que ces signes-là, précisément parce qu'on les reconnaît à cent lieues, ne surgissent pas comme signifiants du processus créateur, mais comme tessères de l'art traditionnel. Dès lors, il devient délicat de parler encore d'idiolecte. Ces formes de l'image sont justement des formes communes, elles sont formes, elles portent les indices de leur propre conclusion, de leur propre mort. Il s'agit d'un effacement (ou d'un refoulement?) de la force motrice pulsionnelle en faveur de ce "retour à l'état antérieur» décrit par Freud, et au cours duquel

[...] cette tendance régressive ne peut viser qu'à rétablir des

formes moins différenciées, moins organisées, ne comportant plus à la limite de différences de niveau énergétique. 17

Lorsque le créateur dépose d'autres éléments, inconnus, décalés, hors champ, on ne peut être assuré que de la pâte qui façonne son environnement, c'est-à- 
dire la médiation de fait, entre le monde et lui: il livre sa mouture, sa version, son tissage. Cela ne veut pas dire que tout ce qui jusque-là fut inconnu puisse être considéré comme de l'art; il est nécessaire d'analyser de très près ce qui nécessite le concept d'art pour être, mais cela ne veut pas dire non plus que toute ouvre utilisant le cadre, les couleurs, etc., ne puisse pas appartenir à l'art contemporain. Tout est une question de distanciation par rapport à l'interprétation de l'icône. Dans le monde de l'art, l'icône est très souvent interprétée comme un symbole et, à ce titre, elle doit se répéter en toute liberté. Cette confusion est génératrice de catastrophes esthétiques. En effet, lorsque l'un est mis pour l'autre, il n'y a plus d'accueil possible pour la nouveauté. Tout élément non conforme au registre des signes typifiés par le système artistique est rejeté par lui, non reconnu. C'est sans doute pour les mêmes raisons que l'art est souvent défini par des acteurs qui ne sont pas les créateurs (directeurs, responsables de galeries et autres). L'impression même d'être tenu devant de l'art «décrété» montre que les signes habituels ne sont pas présents et que la vacuité de la signification d'un signe non codé ne répond pas aux exigences d'un certain seuil de l'imitation. Le processus d'imitation, compris dans ses plus larges effets, est une véritable mutilation de l'art, «inhibiteur» de la création, conditionneur du musement (le musement ne peut être autre chose que ce que l'on est). Il est avant tout - non en lui-même, mais par les effets qu'il produit lorsqu'on le réduit à un obligatoire saisissement de repères esthétiques - le geôlier de l'idiolecte, le gardien vigilant du système symbolique, grâce auquel toute œuvre produite aura quelque chose appartenant au système, le référençant implicitement aux conventions interprétatives possibles. Si le ton peut paraitre polémique, il témoigne aussi d'une certaine usure, en ce que, un siècle après les gestes iconoclastes de Marcel Duchamp, l'art contemporain est diabolisé, massacré et si médiocrement accueilli par le grand public. Il me semble qu'il y a là une impossibilité absolue à la fonction d'accueil, non pas pour applaudir à la première fantaisie ou à la première rouerie esthétique, mais pour rendre possible l'inscription esthétique, celle qui, justement travaillée par le musement et la contrainte physique sociale, fait de l'œuvre leur prolongement.

Cet espace précieux, apprivoisé dès le plus jeune âge, est resté continu pour l'artiste, il fait partie de ce que M. Balat a fini par nommer le corps sémiotique. Lorsque l'artiste dépose ces signes, là ou ailleurs (atelier ou montagne, plage ou galerie), ce ne sont que des tessères de l'art dont on peut supposer, pour maintenir l'hypothèse analytique, que la mère serait l'ensemble des œuvres, des critiques, de ce que la société réalise, devance ou expie jusqu'à ce jour - société dont je fais partie et dont je laisse aujourd'hui une trace. L'inscription ira au-delà, elle nécessitera le relais institutionnel ou éclairé, pour Être. En ce sens, l'espace de l'idiolecte requiert accueil et vigilance et adopte d'innombrables apparences singulières. L'œuvre déposée par l'artiste répond au principe de plaisir en ne s'opposant pas ici au principe de réalité dont elle est la condition sine qua non; et la préservation de sa singularité se fait au prix d'une individualisation non pas du créateur mais du public ${ }^{18}$ !

On pourra, pour conclure, dire que l'idiolecte plastique est un produit du cadre dont l'expression ne peut être que hors champ, tout comme le langage est un produit d'une société originaire dont la parole créatrice est hors champ de la convention, et tout comme encore le temps de la séance analytique est pétri par un ensemble de faits, impressions et jugements, qui contribuent au temps plus englobant de la cure. 


\section{NOTES}

1. L'idée de "pairs de l'objet" convient mieux à mon sens que celle de consensus, liée à des politiques d'assimilation à l'art, trop rapide, bâclée parfois. Il vaudrait mieux en effet qu'un objet ne soit pas trop vite reconnu par son domaine de prédilection pour lui laisser le temps de l'ambiguité, pendant lequel il est un objet "vague", afin de trouver le meilleur champ possible d'interprétation.

2. Il s'agit de la production de minuscules gouttelettes de salive en suspension dans l'air émises dès que nous parlons, lors de la respiration, de la toux, des éternuements, etc. Son volume dépend du débit de ventilation et crée une sorte d'auréole de buée invisible, souvent la cause de transmission bactérielle. Cf. H. Hermann et J.F. Cier, 1970: 430. Pour une description plus simple, voir le site Internet http://www.med.univ-rennes 1.fr/resped/s/viro/omyxo/orthomyx.htm.

3. On peut notamment consulter à ce sujet les œuvres Goya et son médecin Arrieta (1820), malgré que les personnages se tiennent l'un et l'autre, et Portrait de Manuel Godoy (1801), où le premier personnage d'Espagne ne peut en écouter un autre qu'en prêtant l'oreille sans jamais faire face à son interlocuteur, c'est-à-dire sans rapprocher leurs profils. Mais si Goya peut être exemplaire à cause de ses transgressions possibles, on peut trouver d'autres peintres qui ont aussi mis à distance leurs personnages. Fragonard, dans Le Verrou (1780), tord la posture de la femme, qui du même coup masque la bouche de son amant malgré la proximité des visages. Dans la peinture de Konrad Witz ( $\mathrm{La}$ Rencontre d'Anne et de Joachim à la Porte dorée, 1446), le ton de la confidence évoqué n'implique pas le rapprochement des têtes, mais au contraire colle les corps séparés par l'étoffe du vêtement et écarte ainsi les visages, jusqu'à d'ailleurs faire croiser leurs regards hors d'un face-àface. La peinture est une source intarissable pour cette question.

4. Dans la théorie peircienne du signe, la sémiose est le signe-action à partir duquel le processus d'interprétation est déclenché: «action ou influence qui est ou implique la coopération de trois sujets, tels qu'un signe [au sens de representamen], son objet et son interprétant, cette influence tri-relative n'étant en aucune façon réductible à des actions entre paires». (5-484; Peirce, $1978: 133)$.

5. En 1991, j'ai opéré une distinction dans la définition de la sémiose en dichotomisant les signes-actions selon le contexte. Il y a donc trois formes de sémioses: la sémiose de connaissance, la sémiose de création et la sémiose dégénérée. F. Caruana, 1991: 16.

6. J'ai réalisé une série de bâtons de deuil pour l'exposition d'art sacré en 2001 (salle des archives à Perpignan), constitués de bois, d'os, de ruban, de coquillages, etc.

7. Le concept de tessères corporelles a été développé en psychanalyse par Michel Balat. Il s'agit d'un signe d'incorporation d'un type, repérable dans la "prédisposition" physique à révéler un trait. Il faut en cela, et pour une meilleure saisie du concept, voir dans quel contexte il est énoncé. M. Balat, 1998: 18-19; $2000: 126$.

8. Ces propos de Beuys sont cités par W. Schade dans le texte du catalogue Joseph Beuys, Premières aquarelles. Schade renvoie ici à un entretien publié dans V. Harlan, Was ist Kunst, Werkstattgespräch mit Benys, Stuttgart, 1986, p. 66, note 1.

9. Settis appelle l'ensemble de ces propositions «l'atelier exégétique», au cours duquel il montre bien que les interprétations proposées étaient réellement possibles et que celle qu'il y ajoute l'est d'autant plus qu'elle appartient à un schéma iconographique ignoré par tous. En effet, Settis propose de considérer l'œuvre par rapport à un même schéma iconographique, alors que les exégèses énoncées attribuent à Giorgione «l'invention de la scène». S. Settis, 1987: 90.

10. Je fais référence au travail de Michel Balat avec l'équipe de
Château Rauzé dirigée par Edwige Richer, qui consiste depuis une dizaine d'années à faire l'hypothèse du musement chez les personnes en phase végétative (CHU de Bordeaux).

11. Je fais référence au texte intitulé «Un argument négligé en faveur de la réalité de Dieu " (G. Deledalle, 1990: 180).

12. La secondéité relève d'une catégorie générale, avec la priméité et la tierciété qui sont les catégories des formes de l'expérience. La secondéité phénoménologique est celle qui s'impose à moi lorsque je me cogne à la table (idéoscopie, classement et description des idées de l'expérience ordinaire et phanéroscopie, classement des catégories du phénomène).

13. Le parallèle établi entre ce que l'artiste produit et la difficulté d'interprétation de ce à quoi l'œuvre donne lieu, tout comme l'est l'écriture des personnes en phase végétative, s'ajuste à l'argument négligé en faveur de la réalité de Dieu. Si nous ne pouvons en fournir la preuve, le processus lui est à l'œuvre. Il ne s'agit plus de croyance, ni d'existence mais de réalité. En ce sens, ce qui relève de l'art est une perpétuelle mentalisation du monde. G. Deledalle, 1990: 180.

14. Il s'agit de la $3 e$ partie consacrée à la réalité de Dieu et plus particulièrement à la notion de "plausibilité». Ibid., p. 181.

15. J. Lacan, 1981: 225.

16. Un type, ou légisigne, désigne en sémiotique tout signe conventionnel. «Le légisigne n'est pas un objet singulier, mais un type général qui [...] doit être signifiant. Tout légisigne signifie par son application dans un cas particulier, qu'on peut appeler sa réplique : par exemple, le mot "the" qui apparaît d'ordinaire en anglais quinze à vingt-cinq fois par page». Peirce, 1978: 139.

17. Vocabulaire de la psychanalyse de J. Laplanche et J.-B. Pontalis, sous la dir. de D. Lagache, 11e éd., Paris, P.U.F., 1992, p. 379.

18. De ce point de vue, je rejoins Levi-Strauss, en paraphrasant ce qu'il énonce à propos de la relation entre "art primitif» et art moderne: "[...] avec l'art des temps modernes, il s'agirait donc d'une individualisation croissante, non pas du créateur mais de la clientèle. Ce n'est plus le groupe dans son ensemble qui attend qu'il fournisse certains objets façonnés selon des canons prescrits, mais des amateurs - aussi bizarre que le terme puisse paraitre, dans une comparaison avec des sociétés très différentes de la nôtre - ou des groupes d'amateurs ». C. Levi-Strauss, 1969: 69-70.

\section{RÉFÉRENCES BIBLIOGRAPHIQUES}

BALAT, M. [2000]: Psychanalyse, logique, éveil de coma: le musement du scribe, Paris, L'Harmattan;

[1998] (sous la dir. de ): Autisme et éveil de coma (Actes du colloque de 1997), Nîmes, Théétète-Le Champ social.

CARUANA, F. [1991]: Problèmes d'interprétation en peinture, thèse de doctorat, Perpignan.

DeledAlle, G. [1990]: Lire Peirce aujourd'hui, Bruxelles, Éd. de Boeck, coll. «Le point philosophique».

FRIED, M. [2000]: Le Modernisme de Manet, Paris, Gallimard.

Hermann, H. et J.-F. Cier [1970]: Précis de physiologie, Paris, Éd. Masson, 1970, p. 430.

LACAN, J. [1981]: Séminaire III. Les Psychoses, texte établi par J.-A. Miller, Paris, Seuil.

LeVI-STRAUSS, C. [1969]: Entretiens avec G. Charbonnier, Paris, 10/18.

PEIRCE, C.S. [1978] : Écrits sur le signe, rassemblés, traduits et commentés par G. Deledalle, Paris, Seuil. SETTIS, S. [1987]: L'Invention d'un tableau, trad. par O. Christin, Paris, Minuit, coll. «Le sens commun». 